\title{
Enabling the Adoption of Wearable Computers in Enterprises - Results of Analyzing Influencing Factors and Challenges in the Industrial Sector
}

\author{
Sebastian Hobert \\ University of Goettingen \\ shobert@uni-goettingen.de
}

\author{
Matthias Schumann \\ University of Goettingen \\ mschuma1@uni-goettingen.de
}

\begin{abstract}
Wearable computers like smart glasses or smartwatches enable the use of information systems in application scenarios in which information technology has rarely been used until now. The reason for this is, that users are able to interact with the devices handsfree, e.g. by using voice commands. A hands-free use is in particular relevant for enterprises in the industrial sector, as industrial workers often need to perform tasks manually, e.g. in manufacturing or maintenance. However, the technology is currently not used widely in enterprises. Thus, the aim of our research is to identify influencing factors and related challenges of using wearable computers in order to analyze how its adoption can be increased. Based on an empirical interview study within the industrial sector, we identified 11 influencing factors and 25 related challenges which affect the adoption of wearable computers.
\end{abstract}

\section{Introduction}

For several years increasing efforts to develop market-able wearable computers (like smart glasses or smartwatches) have been seen [16]. Even though many different smartwatches and smart glasses are now available for consumers, they are currently rarely used in enterprises. Particularly in the industrial sector, the use of wearable computers is not widespread until now. However, especially when employees need to work with both hands (e.g. in order to assemble a workpiece), wearable computers can be useful to assist them [21, 23]. A domain expert who participated in our study summarized this as follows:

"Every time when you need to work with your hands and need additional information, wearables are at an advantage." (Expert 3)

Application scenarios in which employees can be supported by wearable computers exist in many different areas in the industrial sector [10]: For instance, smart glasses can be used to guide employees through manufacturing processes by displaying stepby-step instructions in the workers' field of vision. Another exemplary scenario which promises savings in cost and time is to use smart glasses for remote support to assist local workers to solve problems [17]. In this case, smart glasses can be used to record the worker's field of vision while he is trying to solve an error and stream the video live to a remote expert. Based on this live stream, the expert is able to assist the local worker in real-time.

However, as smart glasses and other wearable computers are not yet in use in most industrial companies, it needs to be analyzed how the adoption of wearable computers can be promoted. Therefore, the aim of our study presented in this research paper is to analyze challenges and identify factors which influence the adoption and use of wearable computers. Even though our interview study focuses on using wearable computers in the industrial sector, most influencing factors and related challenges can be easily transferred to companies within different sectors.

In order to describe our research targets, we ask the following research questions:

RQ1: Which factors influence the adoption of wearable computers in enterprises in the industrial sector?

RQ2: What challenges impede the successful use of wearable computers in industrial enterprises?

To answer these research questions, the remainder of this article is structured as follows: First we present definitions of basic terms like wearable computers and outline the theoretical background of technology adoption by focusing on the Technology-OrganizationEnvironment Framework by Tornatzky and Fleischer [25]. Afterwards, we describe our research methodology. Following this methodology, we show the findings of our study by describing both influencing factors and related challenges. Finally, we discuss the results and present future research directions in the conclusion. 


\section{Basics and theoretical background}

\subsection{Wearable Computers}

Wearable computers can be defined as computing devices which are worn on the user's body $[4,12,20]$. Typical examples of currently available and marketable wearable computers are smart glasses which are worn on the user's head and smartwatches which are worn on the user's wrist. Usually wearable computers are always turned on while users are wearing them [20]. Therefore, they are always available to the user independently of a specific time or location.

Technically, wearable computers can be seen as an enhancement of smartphones and tablets because they share mostly the same internal hardware components like integrated processors or wireless communication technologies [8, 19]. However, in contrast to mobile devices wearable computers can be used hands-free as they are worn on the body and don't need to be hold in the user's hands. Because of this advantage, wearable computers don't need the user's focus and can be used incidentally. Thus, wearable computers can proactively start interaction with the user, e.g. by displaying information in the user's field of vision [3]. In order to fully profit from the hands-free use and to enable interaction with the user, wearable computers usually provide several input and output capabilities:

For instance, smart glasses have integrated headmounted displays which are located directly in the user's field of vision [11]. Furthermore, they often provide audio output via integrated speakers (e.g. bone conduction transducers) or headphones. Besides voice input with speech recognition [6], smart glasses often have built-in sensors like GPS or a gyroscope in order to retrieve input from the environmental surrounding. Some smart glasses even have a camera built-in in order to capture photos and videos. Finally, most smart glasses provide limited touch input via buttons or a touchpad.

The input and output capabilities of other types of wearable computers like smartwatches are similar, but usually they are more limited [5]: The output capabilities of smartwatches are restricted to a small display and sometimes to audio output via Bluetooth headphones. The input of user data is often done via voice as the small size of touch screens is only suited for simple inputs (like selecting a predefined menu option).

Wearable computers have been targeted in prior research for more than 50 years $[13,24]$. The existing research mainly focuses on technical aspects of wearable computers like building hardware devices or analyzing sensory data of wearable computers.
However, research about the actual use of wearable computers in enterprises (or especially in the industrial sector) is limited. Only in few cases some specific application scenarios of the industrial sector have been analyzed. For instance, Paelke [18] analyzed how workers can be supported using augmented reality and Guo et al. [9] showed how picking processes in logistics can be simplified using head-up displays for employees. Even though there exists some research about the use of wearable computers in enterprises and the industrial sector, the adoption of wearable computers in enterprises has not been covered yet.

\subsection{Theoretical Background}

In prior research, the organization-level theory Technology-Organization-Environment Framework (TOE framework; see Figure 1) of Tornatzky and Fleischer [25] has often been used to explain the adoption and implementation of new technologies in enterprises [2].

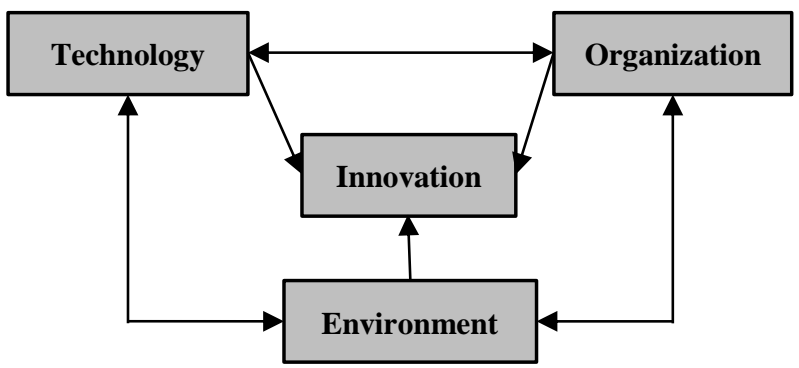

\section{Figure 1. Technology-Organization-Environ- ment Framework [2, 25]}

Numerous studies have applied this framework in IS research in order to explain which influencing factors (like surrounding conditions) affect the adoption of new technologies: For instance, Doolin and Ali [7] studied the adoption of mobile commerce technology for supply chain activities using semistructured interviews. For analyzing the results, they classified technological attributes, organizational characteristics and environmental conditions according to the TOE framework. Another example which is related to the industrial sector is a study presented by Angeles [1] in which the deployment of RFID systems has been analyzed using the TOE framework. Summarizing these prior studies, it has been shown that the TOE framework is suitable for analyzing the adoption of new technologies. 


\section{Methodology}

In order to identify relevant factors which influence the adoption of wearable computers in the industrial sector, we chose to conduct explorative interviews with domain experts. Based on this empirical data, we pursued the aim to derive relevant influencing factors for enterprises in general and for the industrial sector in particular.

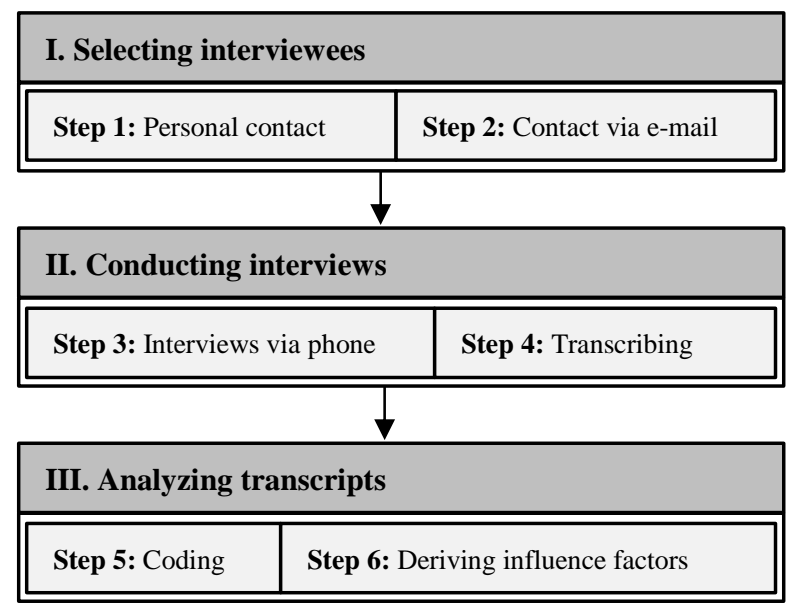

Figure 2. Research design

As displayed in Figure 2, our methodological approach was divided into three parts. First, we selected potential domain experts from enterprises in the industrial sector which have either experience with using wearable computers or are providing wearable solutions for industrial companies. To this end we contacted 22 interviewees during an industrial fair in April 2015. To increase our sample size, we identified further companies which could possibly provide insights about using wearable computers in industries from an internet search. Based on this, we increased our sample size by 34 . In total we contacted 56 domain experts. As 21 domain experts accepted our interview invitation, we had an acceptance rate of $37.5 \%$.

After finishing the interviewees' selection, we conducted our interviewees via phone starting in July 2015. In order to focus on our aim to identify influencing factors and related challenges, we prepared an interview guideline. However, as we also wanted to leave the interviewees enough room to express own ideas, the guideline was semi-structured [15]. To allow in-depth analysis of our interviews, we asked all domain experts whether we were allowed to record the interviews on tape and to transcribe them afterwards. 19 out of 21 interviewees were recorded and transcribed with an average of $42 \mathrm{~min}$ per interview. Only 2 experts denied the recording, here we collected data by intensive note taking. The characteristics of our conducted interviews are displayed in Table 1.

\section{Table 1. Characteristics of conducted} interviews

\begin{tabular}{|c|c|c|}
\hline No. & Function & Rec. \\
\hline \multicolumn{3}{|c|}{ July 2015} \\
\hline Exp1 & IT Product Manager & $\checkmark$ \\
\hline \multicolumn{3}{|c|}{ August 2015} \\
\hline Exp2 & $\mathrm{CEO}$ & $\checkmark$ \\
\hline Exp3 & $\mathrm{CEO}$ & $\checkmark$ \\
\hline \multicolumn{3}{|c|}{ September 2015} \\
\hline Exp4 & CTO & $\checkmark$ \\
\hline Exp5 & $\mathrm{CEO}$ & $\checkmark$ \\
\hline Exp6 & $\mathrm{CEO}$ & $\checkmark$ \\
\hline Exp7 & $\mathrm{CEO}$ & $x$ \\
\hline Exp8 & IT Solution Architect & $\checkmark$ \\
\hline \multicolumn{3}{|c|}{ October 2015} \\
\hline Exp9 & Head of process management & $\checkmark$ \\
\hline Exp10 & Training Supervisor & $\checkmark$ \\
\hline Exp11 & Field Representative & $\checkmark$ \\
\hline Exp12 & IT Senior Consultant & $x$ \\
\hline \multicolumn{3}{|c|}{ November 2015} \\
\hline Exp13 & $\mathrm{CEO}$ & $\checkmark$ \\
\hline Exp14 & $\mathrm{CEO}$ & $\checkmark$ \\
\hline Exp15 & R\&D Employee & $\checkmark$ \\
\hline Exp16 & Manufacturing IT Employee & $\checkmark$ \\
\hline Exp17 & CTO & $\checkmark$ \\
\hline \multicolumn{3}{|c|}{ December 2015} \\
\hline Exp18 & Logistics Consultant & $\checkmark$ \\
\hline Exp19 & System Analyst & $\checkmark$ \\
\hline Exp20 & $\begin{array}{c}\text { Senior Manager Corporate } \\
\text { Production }\end{array}$ & $\checkmark$ \\
\hline Exp21 & IT Business Analyst & $\checkmark$ \\
\hline \multicolumn{3}{|c|}{$\begin{array}{l}\checkmark: \text { Recording of interview was permitted } \\
\text { x: Recording of interview was denied; intensive note } \\
\text { taking to capture content }\end{array}$} \\
\hline
\end{tabular}

After finishing the data collection in December 2015, we analyzed our data using the structured content analysis approach [14] by coding relevant statements of the transcribed interviews using open and selective coding. To minimize coding errors and subjective influences, two persons were involved in the coding process. This resulted in 398 relevant statements which we grouped into similar challenges (see section 4). Based on this, we derived influencing factors and classified them according to the dimension of the TOE framework (see section 2.2). As not all interviews were conducted in English, we applied the constant contextual comparison method [22] to translate relevant quotations for publishing the results in this paper to English. 


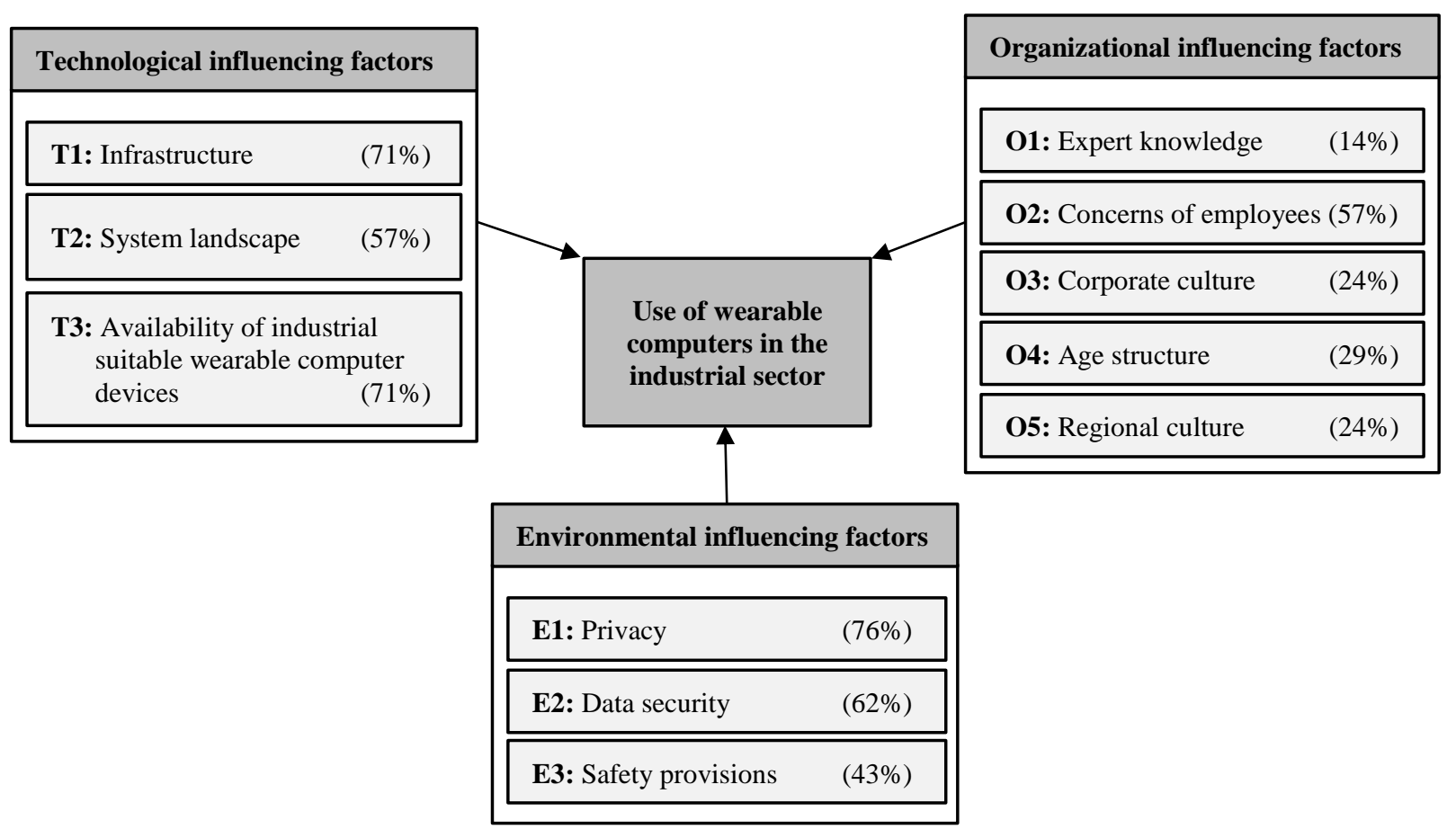

Figure 3. Overview of identified factors

\section{Findings}

As a result of our interview study, we were able to derive 11 influencing factors which correspond to the adoption of wearable computers in the industrial sector. We classified them as technological, organizational and environmental influencing factors according to the Technology-OrganizationEnvironment Framework of Tornatzky and Fleischer [25] (see section 2.2). An overview of the derived influencing factors (including the percentage of interviewees naming the factors) is depicted in Figure 3 . In the following, we describe these influencing factors and present 25 related challenges.

\subsection{Technological influencing factors}

Based on our empirical study, we identified three technological influencing factors. These factors represent characteristics of all technologies which are already present in enterprises as well as available and market-able computing systems which are not currently in use [2]. The first aspect which influences the use of wearable computers in enterprises, especially in the industrial sector, is the existing technical infrastructure (T1) which was named by $71 \%$ of our interviewees. In particular, the domain experts specified that a complete WiFi coverage is required in order to use wearable computers in almost all cases. Otherwise communication between wearable computer devices and information systems would not be possible. Today in many enterprises outside the industrial sector, the WiFi coverage should not be a problem. However, especially in industrial production facilities the availability of a complete WiFi coverage is not common $\left(\mathrm{T}_{\mathrm{C}} 1.1\right)$. Furthermore, our interviewees stated that even if WiFi is theoretically available in the factory, it often gets disturbed by metal or steel. Additionally, in many cases only few production machines (like assembly robots) are allowed to connect to it, because of security regulations.

Depending on the specific application scenario, an additional challenge is the existence of a broadband internet connection $\left(\mathrm{T}_{\mathrm{C}} 1.2\right)$. An example which was named by our interviewees and requires a broadband internet connection is live remote support via video telephony using smart glasses. Even though in most developed countries this shouldn't be a problem, many industrial production facilities are located in countries where a broadband internet connection cannot always be guaranteed. Table 2 depicts exemplary quotations describing this technological influencing factor and summarizes the identified challenges. 
Table 2. Technological influencing factor (1/3)

\begin{tabular}{|l|l|}
\hline \multicolumn{2}{|c|}{ T1: Infrastructure } \\
\hline \multicolumn{2}{|c|}{ "In our company, every single production facility is } \\
connected to the internet. Sometimes the shop floor \\
is not covered completely via WiFi, but it will be \\
done in the future. In principle, we will be able to \\
establish an internet connection [with wearable \\
computers] everywhere. However, the quality may \\
vary." Exp20
\end{tabular}

In addition to the infrastructure, the remaining system landscape (T2) usually consisting of multiple information systems (like enterprise resource planning systems or warehouse management systems) was named as a technical influencing factor by 12 interviewees.

Table 3. Technological influencing factor $(2 / 3)$ T2: System landscape

"There is no uniformed bus system [to access information], no uniformed protocol and no uniform standardization. Everything what is done is the smallest possible compromise." Exp11

"What is the operating system of the smart glass? [...] you need to check if it can be integrated in the system landscape. Every company has guidelines regarding the IT system landscape [...] Then you need to check if it is possible to integrate [wearable computers] and you need to check if they disturb other systems." Exp15

$\mathbf{T}_{\mathbf{C}}$ 2.1: Integration of wearable computers in existing system landscape

$\mathbf{T}_{\mathbf{C}}$ 2.2: No standardized interfaces for accessing arbitrary information

$\mathbf{T}_{\mathbf{C}}$ 2.3: Dependence on vendors

According to them (see Table 3), the aim of using wearable computers in the industrial sector is to support the employees. There it is necessary to integrate the wearable computer devices in the existing system landscape $\left(\mathrm{T}_{\mathrm{C}} 2.1\right)$. For instance, wearable computers need access to contextual information like sensor data from machineries or picking lists from warehouse management systems to support employees during their work. Consequently, interfaces to relevant information systems are required to fetch that information. However, as of today there are no standardized interfaces available which can be used to fetch arbitrary information $\left(T_{C} 2.2\right)$. In fact, usually it is required to use multiple, technically different interfaces to access information from information systems and machineries. Furthermore, market-able wearable computer applications are often designed to work only with information systems from selected vendors $\left(\mathrm{T}_{\mathrm{C}} 2.3\right)$.

Finally, 15 interviewed domain experts stated that the availability of industrial suitable wearable computer devices (T3) influences the adoption (see Table 4). According to the interviewees, wearable computer devices are not yet widely used in industrial enterprises and need to be purchased in advance $\left(\mathrm{T}_{\mathrm{C}} 3.1\right)$. Even though multiple market-able smart glasses and smartwatches are available for purchase from different vendors, most domain experts highlighted that it is often not possible to buy appropriate devices which are suited for being used in rough environments like in industrial production facilities $\left(\mathrm{T}_{\mathrm{C}} 3.2\right)$. Most market-able wearable computers are designed for consumers and are therefore less robust. Important certifications of conformity which are needed for a professional use in industries are often missing. Furthermore, many available wearable computers are not suitable for supporting employees during their daily work as the hardware is often technically restricted $\left(\mathrm{T}_{\mathrm{C}} 3.3\right)$. For instance, many devices do not have enough battery capacity to display information for a typical working shift of eight hours or overheat if they are used permanently.

Table 4. Technological influencing factor $(3 / 3)$ T3: Availability of industrial suitable wearable computer devices

"Smart glasses are not so robust [...] they should not fall off, otherwise they are broken" Exp21

"Currently available devices are very fragile at least when I am using them in the industrial sector, because you are not working in a clean environment." Exp20

"The development of smart glasses focuses on the consumer market and not on working shifts of the industrial sector $[\ldots]$ e.g. the battery needs to be separated from the wearable computer $[\ldots]$ because of the heat generation" Exp18

TC 3.1: No suitable wearable computers available for industrial enterprises

$\mathbf{T}_{\mathbf{C}}$ 3.2: Wearable computers are not designed for being used in rough environments

$\mathbf{T}_{\mathrm{C}}$ 3.3: Technical restrictions of wearable computers 


\subsection{Organizational influencing factors}

Organizational influencing factors describe aspects which refer to the characteristics and structure of the analyzed enterprises [2]. In this category, we identified five influencing factors and our interviewees described more challenges than in any other category.

The first critical influencing factor which was mentioned by $14 \%$ of our interviewees is the need for expert knowledge (O1) in order to be able to introduce wearable computing devices in enterprises and to use them in day-to-day business (see Table 5). As the wearable computing technology is rather new and has not been used in industries before, most interviewed experts stated that there is no adequate knowledge available in their company $\left(\mathrm{O}_{\mathrm{C}} 1.1\right)$. In particular, even if modern and technically similar devices like smartphones and tablets have been used in industries for many years, the interviewees claimed that they need support from experienced third parties to introduce wearable computers in their day-to-day business. However, several experts stated that it was hard to find experienced third party developers and consultants $\left(\mathrm{O}_{\mathrm{C}} 1.2\right)$. In some cases, several enterprises even needed to build up knowledge on their own before starting pilot projects.

Table 5. Organizational influencing factor $(1 / 5)$ 01: Expert knowledge

"Another important aspect is that we do not have any computer scientists or software engineers which are able to improve the [wearable computer] application. [...] Currently, we are not able to [change the software] without the help of third parties. [...] We always rely on external partners." Exp9

O 1.1: Lack of knowledge about wearable computers within industrial companies

OC1.2: Few experienced third party developers and consultants available

While starting first pilot projects or planning to introduce wearable computer applications 12 interviewees were confronted with concerns of employees (O2). The concerns of employees mainly encompass the fear of being watched or surveilled during their work $\left(\mathrm{O}_{\mathrm{C}} 2.1\right)$. For instance, one interviewee explained, that employees in logistics which should be supported by smart glasses to find the right shelf assume to be surveilled by the wearable computers. Because of this fear, some employees denied to use smart glasses. According to our interviewees, further employees refuse to use the technology as they fear job loss $\left(\mathrm{O}_{\mathrm{C}} 2.2\right)$ because they suspect that wearable computers can help to rationalize workflows. In many cases, concerns of employees were communicated by the workers' council. Several interviewees stated that this most often results in a need to negotiate company agreements before it was allowed to test or use wearable computers in the factory. This process was described by several experts as annoying as it slows down the process of starting a pilot project or introducing wearable computer technology massively $\left(\mathrm{O}_{\mathrm{C}} 2.3\right)$. Table 6 depicts exemplary quotations and related challenges.

Table 6. Organizational influencing factor $(2 / 5)$ O2: Concerns of employees

"[...] employees have in mind that they could be surveilled." Exp19

"If anyone [is able to do the work] using smart glasses which guides him, then you do not need me as an expert anymore. Then [the company] will hire someone else who is cheaper.' Those are common prejudices." Exp10

"The workers' council slows down the process [...] because of special requirements that needed to be fulfilled." Exp15

O 2.1: Fear of being surveilled during their work

$\mathbf{O}_{\mathrm{C}}$ 2.2: Fear of loss of jobs

OC2.3: Negotiations with workers' council slow down process of introducing wearable computers

According to five interviewees, the willingness to introduce new technologies like wearable computers is further influenced by the corporate culture $(\mathbf{O 3})$ and therefore depending on both, the management $\left(\mathrm{O}_{\mathrm{C}} 3.1\right)$ and the employees who work in the operating departments $\left(\mathrm{O}_{\mathrm{C}} 3.2\right.$; see Table 7$)$. The interviewees stated that it was easier to introduce wearable computing technologies if the top management is open to innovative technologies in general and to wearable computers in particular. A lack of management support was otherwise named as a blocking factor which could complicate or even prevent the use of wearable computers. In addition to gain support from the management our interviewees stated that it is a challenge to convince employees from operating departments to support the introduction of new technologies like wearable computers. It was pointed out by several interviewees, that this is especially relevant if wearable computers should be introduced in production facilities as their corporate culture is often rather conservative $\left(\mathrm{O}_{\mathrm{C}} 3.3\right)$ : Using new technologies is often seen as a potential risk which could disturb production processes. 
Table 7. Organizational influencing factor (3/5) O3: Corporate culture

"Depending on the size of the production facility, we have multiple management levels. [...] they do not understand what is the advantage and why

$\oiiint$ [wearable computers] are useful. Because of this, it - is hard for them to accept [the new technology]." Exp20

"Employees in production are often averse to new technologies, tools or changes. If anything goes wrong than it is very expensive. That's the reason why they are averse to innovative products." Exp16

$\mathbf{O}_{\mathrm{C}}$ 3.1: Lack of management support

$\mathbf{O}_{\mathbf{C}}$ 3.2: Lack of support of employees from operating departments

O 3 3.3: Common conservative attitude in production facilities

In addition to that, the acceptance of the wearable computers by the actual workers was stated as depended of the age structure (O4) of the employees (see Table 8). As mentioned by $29 \%$ of the interviewed experts, especially young employees are more curious to try smart glasses and smartwatches and to adopt them finally. In contrast to that, older employees are often more skeptical about using wearable computers $\left(\mathrm{O}_{\mathrm{C}} 4.1\right)$. However, several experts mentioned that after trying smart glasses for several hours, often also older employees accepted the wearable computers if - and only if - they obtain a real advantage (like getting guidance by the wearable computer). While younger employees adopt innovative technology easier, older employees often need to be convinced $\left(\mathrm{O}_{\mathrm{C}} 4.2\right)$.

Table 8. Organizational influencing factor $(4 / 5)$

\begin{tabular}{|c|c|}
\hline \multicolumn{2}{|r|}{ 04: Age structure } \\
\hline & $\begin{array}{l}\text { "So the tendency [among our employees] was: } \\
\text { younger people are very open and actually excited } \\
\text { about wearables. [...] With the older generation, } \\
\text { they were quite skeptical and also more - I would } \\
\text { not say not accepting - but they are very skeptical } \\
\text { with that." Exp } 21\end{array}$ \\
\hline & $\begin{array}{l}\text { O 4.1: Lower interest of older employees in using } \\
\text { wearable computers } \\
\text { OC4.2: Convincing older employees required }\end{array}$ \\
\hline
\end{tabular}

Finally, the last identified organizational influencing factor is the regional culture (O5). Our interviewed experts recognized differences regarding the adoption of wearable computers depending on the location of the industrial company. Especially if the industrial factory is located in Europe it was more difficult to convince both - employees and management - of the advantages of using wearable computers in industries $\left(\mathrm{O}_{\mathrm{C}} 5.1\right)$. In many other regions like in the US or Asia the adoption of wearable computers was stated as higher. Because of those regional differences, two of our experts stated, that they tried to start pilot projects in Europe but the effort to convince all relevant parties was experienced as too high. Therefore, both pilot projects were started in plants outside Europe. Table 9 summarizes this influencing factor which was mentioned by five interviewees.

Table 9. Organizational influencing factor $(5 / 5)$ O5: Regional culture

"I really would say that there are differences among countries. For instance, companies in Germany and all over Europe are more reserved." Exp3

\section{OC5.1: Openness to use wearable computers} varies regionally

\subsection{Environmental influencing factors}

In the third category, we identified three influencing factors and six related challenges. This category encompasses surrounding conditions like laws or regulatory guidelines which need to be complied by enterprises [25].

The most important factor which externally influences the adoption of wearable computers is privacy (E1) as it was mentioned by $76 \%$ of our interviewees (see Table 10). This aspect was stated as a critical factor that should be taken care of. As wearable computers and especially smart glasses are often able to take pictures and record audio or videos, several interviewees were skeptical whether such devices are allowed by law in their factories as this gives the possibility to surveil employees surreptitiously. According to the interviewees, it is required to restrict unused technical capabilities of devices so that surveillance is not possible anymore $\left(\mathrm{E}_{\mathrm{C}} 1.1\right)$. For instance, if no camera input is required for a use case it needs to be secured, that there is no possibility to take pictures or videos using the smart glasses.

Furthermore, in many companies several competing laws and regulations need to be respected (like the Data Protection Directive in Europe and local regulations). Especially if large industrial companies want to use wearable computers in all production facilities across several countries, multiple local regulations need to be taken care of $\left(\mathrm{E}_{\mathrm{C}} 1.2\right)$. 
Table 10. Environmental influencing factor $(1 / 3)$

\begin{tabular}{|l|l|}
\hline \multicolumn{2}{|l|}{ E1: Privacy } \\
\hline & "I think [using wearable computers] is definitely \\
going to be an issue for privacy [...]" Exp21
\end{tabular}

In addition to privacy, data security (E2) was named as an influencing factor (see Table 11). $57 \%$ of the experts stated that it is important to protect industrial secrets like how to manufacture products. In particular, multiple experts stated that adequate security precautions (like the encryption of all data transfers) are required in order to prevent unauthorized access and data breaches $\left(\mathrm{E}_{\mathrm{C}} 2.1\right)$. Even though Exp16 stated that this aspect is important, he added that it is not a real technical problem to protect data adequately.

Table 11. Environmental influencing factor $(2 / 3)$

\begin{tabular}{|l|l|} 
E2: Data security \\
\hline "If you want to use wearables [...], you are very \\
restricted because of the fear of industrial \\
espionage. Everything needs to be negotiated with \\
the data security department." Exp16
\end{tabular}

Finally, $43 \%$ of the interviewed experts stated that safety provisions (E3) are a relevant factor which affects the use of wearable computers in industries (see Table 12). Especially as wearable computers usually should be worn all day by the employees, it needs to be taken care of that they do not get harmed by using the technology. Thus, several experts stated that they need to proof that using wearable computers does not harm employees $\left(E_{\mathrm{C}} 3.1\right)$. Therefore, they need to provide studies which indicate that employees do not get distracted or stressed by the devices. Furthermore, further laws and regulations define safety measures which need to be followed in the industrial sector like wearing safety glasses to protect the employees' eyes. Our interviewees stated that this can be a challenge especially if smart glasses should be used $\left(\mathrm{E}_{\mathrm{C}} 3.2\right)$. In this case it needs to be secured that both glasses can be worn simultaneously or alternatively that smart glasses can be integrated in existing protective clothing (like a safety helmlet). Finally, the domain experts named the challenge to avoid distraction of workers when information is displayed proactively by wearable computers $\left(\mathrm{E}_{\mathrm{C}} 3.3\right)$. For instance, Exp17 claimed that displaying information in the users' field of vision on smart glasses can lead to inattention which increases the risk of accidents.

\section{Table 12. Environmental influencing factor} $(3 / 3)$

\begin{tabular}{|c|c|}
\hline & \\
\hline 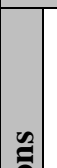 & $\begin{array}{l}\text { "In many areas [in the industrial sector] safety } \\
\text { glasses are required to protect the eyes. Smart } \\
\text { glasses need to fulfill this or need to be integrated in } \\
\text { in such glasses." Exp13 }\end{array}$ \\
\hline & $\begin{array}{l}\text { "Regarding health and hygiene you need to prove in } \\
\text { advance that [wearable computers] do not harm } \\
\text { your employees." Exp15 }\end{array}$ \\
\hline & $\begin{array}{l}\text { "If the employees' field of vision is restricted by } \\
\text { smart glasses then the risk of accidents increases." } \\
\text { Exp17 }\end{array}$ \\
\hline 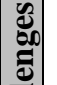 & $\begin{array}{l}\mathbf{E}_{\mathbf{C}} \text { 3.1: Proof required that wearable computers do } \\
\text { not harm employees }\end{array}$ \\
\hline$\overline{\bar{T}}$ & $\mathbf{E}_{\mathbf{C}}$ 3.2: Saf \\
\hline & $\mathbf{E}_{\mathbf{C}} \mathbf{3 . 3}: \quad \mathrm{Av}$ \\
\hline
\end{tabular}

\section{Discussion and implications}

Our findings presented in this study imply that the adoption of wearable computers in industries is influenced by technological, organizational and environmental factors. In total we were able to identify 11 influencing factors and 25 related challenges. In principle all captured challenges are important and need to be targeted when wearable computers should be introduced in industrial companies. However, several experts stated that technological challenges will not be a big problem in practice in the future: Even though there exist some challenges regarding the available infrastructure (T1) or system landscape (T2), all related challenges can be solved. For instance, even if a complete $\mathrm{WiFi}$ coverage is not common in most industrial factories at the moment, all related issues can be solved: Even though the disruption of WiFi signals by steal and metal $\left(\mathrm{T}_{\mathrm{C}} 1.1\right)$ cannot be prevented, the issue can be bypassed by installing additional access points in areas which are currently shielded by steal and metal. Thus, this challenge can be solved by investing into additional technical infrastructure.

Only the industrial suitability of wearable computers (T3) might be still an issue in very rough environments (e.g. in production). However, several 
experts indicated that next generations of wearable computers will certainly solve these issues. Thus, it will only be a matter of time until wearable computers are fully compatible to industrial environments.

In contrast to that, organizational challenges can be seen as more important. This is reflected by the fact, that most challenges ( $44 \%$ ) identified in this study are related to organizational issues. Whereas expert knowledge (O1) will be available in the future more easily as soon as the adoption of wearable computers increases, cultural challenges $(\mathrm{O} 3, \mathrm{O} 5)$ and concerns of employees (O2) seems to be more critical. In particular, in organizations in which workers' councils are more powerful, the employees need to be involved early before wearable computers are actually used in business processes. As the technology of wearable computers is unknown to many employees until now, it should be explained extensively and concerns should be taken seriously - especially if older employees are involved (O4). Exp20 summarized this as follow:

"If you explain your employees in detail what you want to achieve [with wearables], if you explain how they can use it, this isn't a real challenge anymore."

Finally, environmental challenges must be considered. Especially if wearable computers should be used across multiple countries, it needs to be taken care of that all applicable laws and regulations are respected. The interviewees evaluated these challenges as solvable if they are considered in advance of a potential use (e.g. when implementing wearable computer applications).

Summarizing the discussed challenges, the identified organizational issues $\mathrm{O} 2, \mathrm{O} 3$ and $\mathrm{O} 5$ seem to be most critical when wearable computers should be used in industrial enterprises. Particularly, if the technology should be introduced in factories in Europe, our interviewed experts stated that this could be problematic mainly because of powerful workers' councils (O2) and a less technology-friendly regional culture (O5) in Europe compared to the US or Asia. Because of this, some of our interviewees even started pilot projects outside Europe (see O5 in section 4.2) which could result in a competitive disadvantage for European companies in the future.

As with any qualitative interview study, there exist some potential limitations. First, the findings of qualitative studies are mainly dependent on the selection of interviewees. In order to retrieve valid influencing factors and related challenges, we carefully selected a suitable amount of experts from a broad range of industrial enterprises like companies from manufacturing, industrial automation and automotive industries as well as suppliers of industrial-suited wearable computer technologies. Thus, this sample should represent a suitable cross section of experts from the industrial sector. Secondly, different researchers might interpret some of our qualitative data differently, as the analysis of interviews is always subjective. However, in order to prevent subjective influences during analyzing the transcribed interviews, we analyzed the data with great care and tried to minimize subjective influences (see section 3): For instance, we applied the structured content analysis approach and double checked our codes with two persons.

Even though the presented study is focused on enterprises in the industrial sector and may have some limitations, the results - influencing factors and related challenges - seem to be generalizable and transferrable to enterprises of different sectors as well. Only few identified influencing factor and challenges are restricted to the industrial sector: For example, the factor that wearable computer devices can be used in rough environments (T3) usually does not apply in other enterprises (e.g. service companies). However, this generalization should be verified in future research. Furthermore, analyzing correlations between influencing factors could bring additional insights about the technology adoption. Especially as multiple participants of our study stated that they expect technological improvements of wearable computers (e.g. ruggedized smart glasses) in the near future, analyzing correlations could be used to predict implications for the adoption of wearable computers in the future. Finally, it should be analyzed in more detail how the location of (industrial) companies affects its technology-friendliness, because the refusal of new technologies like wearable computers may result in competitive disadvantages.

\section{Conclusion}

In this research paper, we pursued the goal of analyzing the adoption of wearable computers in enterprises by identifying influencing factors (RQ1). We further investigated which related challenges (RQ2) have negative implications on the adoption of wearable computers. As a result of our empirical interview study among 21 domain experts, we identified 11 influencing factors and 25 related challenges in three context categories (technological, organizational and environmental). The presented model which is based on the TechnologyOrganization-Environment Framework can contribute to both, research and practice: Our study will contribute to the understanding of using wearable computers in the industrial sector. Thus, it can be used as a starting point to improve existing hardware 
devices and to develop software solutions for wearable computers which are applicable in the industrial sector. Furthermore, the results may help to improve the adoption of wearable computers in enterprises as it allows to predict and to explain potential challenges.

\section{References}

[1] Angeles, R., "Using the Technology-OrganizationEnvironment Framework and Zuboff's Concepts for Understanding Environmental Sustainability and RFID: Two Case Studies", International Journal of Social, Education, Economics and Management Engineering, 7(11), 2013, pp. 1599-1608.

[2] Baker, J., "The Technology-Organization-Environment Framework", in Information Systems Theory: Explaining and Predicting Our Digital Society, Vol. 1, K.Y. Dwivedi, R.M. Wade, and L.S. Schneberger, Editors. 2012. Springer New York: New York, NY.

[3] Billinghurst, M. and T. Starner, "Wearable devices: New ways to manage information", Computer, 32(1), 1999, pp. 57-64.

[4] Boronowsky, M., O. Herzog, and M. Lawo, "Wearable Computing: Information and Communication Technology Supporting Mobile Workers", it - Information Technology, 50(1), 2008, pp. 30-39.

[5] Chaparro, B.S., J. He, C. Turner, and K. Turner, "Is Touch-Based Text Input Practical for a Smartwatch?", in HCI International 2015 -- Posters' extended abstracts: International Conference, HCI International 2015, Los Angeles, CA, USA, August 2-7, 2015. Proceedings, C. Stephanidis, Editor. 2015. Springer: Cham.

[6] Colaço, A., "Sensor design and interaction techniques for gestural input to smart glasses and mobile devices", in Proceedings of the adjunct publication of the 26th annual ACM symposium on User interface software and technology, S. Izadi, A. Quigley, I. Poupyrev, and T. Igarashi, Editors, St. Andrews, Scotland, United Kingdom. 2013.

[7] Doolin, B. and E.A.H. Ali, "Adoption of mobile technology in the supply chain: an exploratory cross-case analysis", International Journal of E-Business Research, 4(4), 2008, p. 1.

[8] Dvorak, J.L., Moving wearables into the mainstream: Taming the Borg, Springer, New York, 2008.

[9] Guo, A., X. Wu, Z. Shen, T. Starner, H. Baumann, and S. Gilliland, "Order Picking with Head-Up Displays", Computer, 48(6), 2015, pp. 16-24.

[10] Hobert, S. and M. Schumann, "Application Scenarios of Smart Glasses in the Industrial Sector: Results of an Empirical Study Among Domain Experts", i-com - Journal of Interactive Media, 15(2), 2016, pp. 133-143.
[11] Kurze, M. and A. Roselius, "Smart glasses linking real live and social network's contacts by face recognition", in Proceedings of the 2nd Augmented Human International Conference. 2011. ACM: Tokyo, Japan.

[12] Malu, M. and L. Findlater, "Personalized, Wearable Control of a Head-mounted Display for Users with Upper Body Motor Impairments", in Proceedings of the 33rd Annual ACM Conference on Human Factors in Computing Systems. 2015.

[13] Mann, S., "Wearable computing: A first step toward personal imaging", Computer, 30(2), 1997, pp. 25-32.

[14] Mayring, P., "Qualitative Content Analysis", Forum Qualitative Sozialforschung / Forum: Qualitative Social Research, 1(2), 2000, p. 20.

[15] Myers, M.D., Qualitative research in business \& management, 2nd edn., SAGE, London, 2013.

[16] O'Connor, M.C., Smart Glasses Finding Work Across Industries - IOT Journal, 2015.

[17] Oldsberg, P., Field Service Support with Google Glass and WebRTC, Handen, Sverige, 2014.

[18] Paelke, V., "Augmented reality in the smart factory: Supporting workers in an industry 4.0. environment", in 19th IEEE International Converence on Emerging Technology and Factory Automation, A. Grau and H. Martinez, Editors. 2014.

[19] Pousttchi, K., M. Weizmann, and K. Turowski, "Added value-based approach to analyze electronic commerce and mobile commerce business models", in International Conference Management and Technology in the New Enterprise. 2003.

[20] Rhodes, B.J., "The wearable remembrance agent: A system for augmented memory", Personal Technologies, 1(4), 1997, pp. 218-224.

[21] Stocker, A., P. Brandl, R. Michalczuk, and M. Rosenberger, "Mensch-zentrierte IKT-Lösungen in einer Smart Factory", Elektrotechnik \& Informationstechnik, 18(7), 2014, pp. 207-211.

[22] Suh, E.E., S. Kagan, and N. Strumpf, "Cultural competence in qualitative interview methods with Asian immigrants", Journal of Transcultural Nursing, 20(2), 2009, pp. 194-201.

[23] Theis, S., M. Wille, and T. Alexander, "The nexus of human factors in cyber-physical systems", in Proceedings of the 2014 ACM International Symposium on Wearable Computers: Adjunct Program, L. Dunne, T. Martin, and M. Beigl, Editors, Seattle, Washington. 2014.

[24] Thorp, E.O., "The invention of the first wearable computer", Second International Symposium on Wearable Computers, 1998, pp. 4-8.

[25] Tornatzky, L.G. and M. Fleischer, The process of technological innovation, Lexington, 1990. 